\title{
The anaphase promoting complex is required for memory function in mice
}

\author{
Tanja Kuczera, ${ }^{1}$ Roman Manuel Stilling, ${ }^{1}$ Hung-En Hsia, ${ }^{1}$ Sanaz Bahari-Javan, ${ }^{1,4}$ \\ Stefan Irniger, ${ }^{2}$ Kim Nasmyth, ${ }^{3}$ Farahnaz Sananbenesi, ${ }^{1,4}$ and Andre Fischer ${ }^{1,5}$ \\ ${ }^{1}$ Laboratory for Aging and Cognitive Diseases, European Neuroscience Institute, Göttingen D-37077, Germany; ${ }^{2}$ Institute for \\ Microbiology and Genetics, University Goettingen, 37077 Göttingen, Germany; ${ }^{3}$ Department of Biochemistry, University of Oxford, \\ Oxford OX1 3QU, England; ${ }^{4}$ Anxiety Diseases Research Group, Laboratory for Aging and Cognitive Disease, European Neuroscience \\ Institute, Göttingen D-37077, Germany
}

\begin{abstract}
Learning and memory processes critically involve the orchestrated regulation of de novo protein synthesis. On the other hand it has become clear that regulated protein degradation also plays a major role in neuronal plasticity and learning behavior. One of the key pathways mediating protein degradation is proteosomal protein destruction. The anaphase-promoting complex/cyclosome (APC/C) is an E3 ubiquitin ligase that targets proteins for proteosomal degradation by the $26 \mathrm{~S}$ proteasome. While the APC/C is essential for cell cycle progression it is also expressed in postmitotic neurons where it has been implicated with axonal outgrowth and neuronal survival. In this study we addressed the role of APC/C in learning and memory function by generating mice that lack the essential subunit APC2 from excitatory neurons of the adult forebrain. Those animals are viable but exhibit a severe impairment in the ability to extinct fear memories, a process critical for the treatment of anxiety diseases such as phobia or post-traumatic stress disorder. Since deregulated protein degradation and $\mathrm{APC} / \mathrm{C}$ activity has been implicated with neurodegeneration we also analyzed the effect of $A p c 2$ deletion in a mouse model for Alzheimer's disease. In our experimental setting loss of APC2 form principle forebrain neurons did not affect the course of pathology in an Alzheimer's disease mouse model. In conclusion, our data provides genetic evidence that $\mathrm{APC} / \mathrm{C}$ activity in the adult forebrain is required for cognitive function.
\end{abstract}

[Supplemental material is available for this article.]

Memory consolidation is a complex process and the underlying molecular and cellular processes are only beginning to emerge. A better understanding of the mechanisms underlying memory formation is especially important since cognitive dysfunction is a key feature of neuropsychiatric diseases such as Morbus Alzheimer that constitute an increasing emotional and economical burden to our societies (Sananbenesi and Fischer 2009).

A unanimous finding across species is that the formation of long-term memories involves the orchestrated regulation of gene expression. As such it is now commonly accepted that learning initiates differential gene expression and critically depends on de novo protein synthesis (Kandel 2001; Barco et al. 2008; Sananbenesi and Fischer 2009; Peleg et al. 2010). On the other hand, a number of studies clearly demonstrated the importance of protein degradation during cognitive function (Lopez-Salon et al. 2001; Merlo and Romano 2007; Artinian et al. 2008; Mabb and Ehlers 2010). A key pathway of protein degradation in eukaryotic cells is ubiquitin-mediated proteosomal degradation (Ciechanover 2005). The final step of ubiquitination, the attachment of ubiquitin to target proteins, is performed by E3-ubiquitin ligases. The anaphase-promoting complex/cyclosome (APC/C) is an E3-ligase consisting of 12 subunits (Fig. 1A) of which APC2 and APC11 form the catalytic core that is essential for ubiquitination (Peters 2006). APC/C activity is controlled via interaction with the coactivator proteins $\mathrm{CDH} 1$ or CDC20 and is essential for cell cycle progression (Peters 2006; Pesin and

${ }^{5}$ Corresponding author.

E-mail afische2@gwdg.de; fax +49 551-399836.

Article is online at http://www.learnmem.org/cgi/doi/10.1101//m.1998411.
Orr-Weaver 2008). It was therefore surprising when APC/C was detected in postmitotic neurons (Gieffers et al. 1999). Subsequent studies demonstrated a role for APC/C activity in neurodevelopmental processes such as axonal outgrowth in cerebellar granular neurons (Konishi et al. 2004; Yang et al. 2009) and data obtained in Drosophila and C. elegans suggested a role of APC/C in synaptic plasticity (Juo and Kaplan 2004; van Roessel et al. 2004). Interestingly, deregulation of APC/C via down-regulation of CDH1 protein has been implicated with neuronal cell death and its contribution to Alzheimer's disease (Almeida et al. 2005; Maestre et al. 2008) and cognitive decline (Li et al. 2008) has been suggested. As such, there is emerging evidence that APC/C activity is critically involved in cognitive processes and that its dysfunction may also contribute to the pathogenesis of neurological diseases.

In this study we tested the role of APC/C in learning and memory processes directly. To this end, we generated mice that lack the essential APC/C subunit APC2 from excitatory forebrain neurons ( $A p c 2$ conditional knockout mice; $A p c 2 \mathrm{cKO}$ ). We show that $A p c 2$ cKO mice develop normally and exhibit no alterations in basal anxiety, explorative and depressive-like behavior or motor coordination. However, the ability to form spatial memories was reduced in $A p c 2 \mathrm{cKO}$ mice. In addition, $A p c 2 \mathrm{cKO}$ were severely impaired in the extinction of fear memories. Since deregulated APC/C activity has been implicated with neuronal cell death, we also tested a role of APC/C in the pathogenesis of Alzheimer's disease by crossing $A p c 2$ cKO mice to an animal model for amyloid pathology. Loss of APC2 in this model did not affect memory function or amyloid-beta-plaque pathology. In summary, our data shows that APC/C function in postmitotic 
A

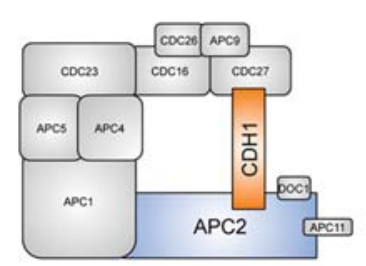

C

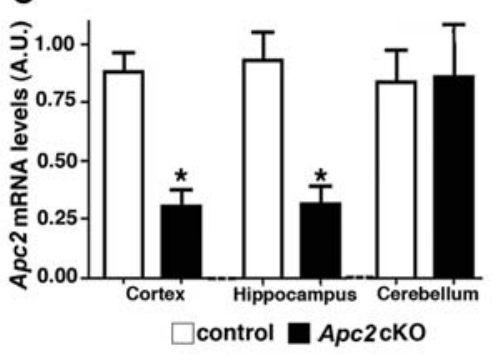

E

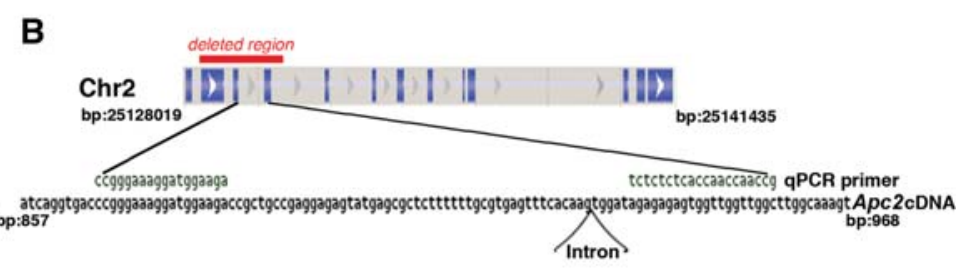

D
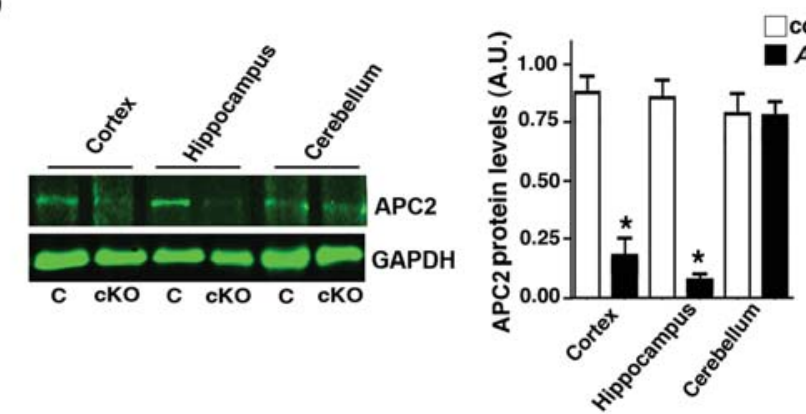

control

Apc2 cKO
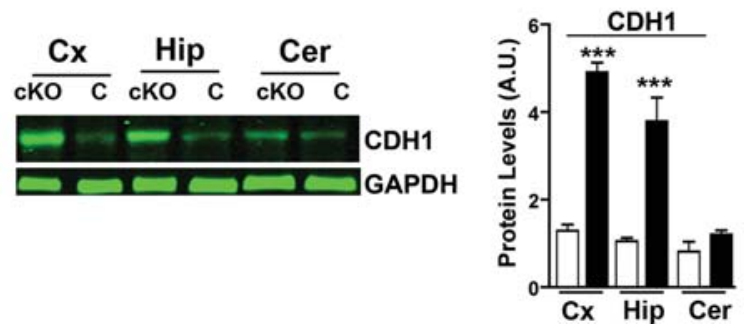

$\square$ control

- Apc2cKO

Figure 1. Deletion of $A p c 2$ from the adult forebrain. $(A)$ Model of the APC/C (Passmore 2005). APC 2 and APC11 form the catalytic core of the APC/C and are therefore essential for its function. $(B)$ The $A p c 2$ gene is localized on chromosome (chr) 2 . In the mice employed in this study exon $2-4$ were flanked by loxP sites. The genomic location of the primers used for qPCR analysis is indicated. (C) The Apc2 mRNA levels are reduced in the forebrain of $A p c 2$ CKO mice $\left({ }^{*} P<0.01\right.$ vs. control). (D) APC2 protein levels are reduced in the forebrain of $A p c 2 \mathrm{cKO}$ mice $\left({ }^{*} P<0.0001 \mathrm{vs}\right.$. control). (Left panel) Representative immunoblot; (right panel) quantification. (E) Elevated Cdh1 levels in the forebrain of $A p c 2 \mathrm{cKO}$ mice (*P<0.001 vs. control). (Left panel) Representative immunoblot; (right panel) quantification. Cx, cortex; Hip, hippocampus; C, control; bp, base pair; AU, arbitrary units. $n=$ 4-6/group. Error bars indicate SEM.

forebrain neurons is critical for spatial memory and the extinction of fear memories in mice.

\section{Results}

\section{Mice lacking APC2 from principal forebrain neurons are viable and show normal brain morphology}

To better understand the role of the APC/C during learning and memory function we decided to delete the essential APC/C subunit APC2 from postmitotic excitatory forebrain neurons of mice (Fig. 1A). To this end we employed animals in which exon 2-4 of the $A p c 2$ gene are flanked by lox P sites (Fig. 1B; Wirth et al. 2004) and crossed them to mice expressing cre recombinase under the control of the CamKII $\alpha$ promoter (Minichiello et al. 1999). As a result we obtained mice bearing the floxed $A p c 2$ allele $\left(A p c 2^{\mathrm{f} / \mathrm{f}}\right)$ in homozygosity and the transgene of CamKII $\alpha$-Cre, which drives the expression of cre recombinase in postmitotic neurons of the adult forebrain, hereafter referred to as $A p c 2$ conditional knockout mice $(A p c 2 \mathrm{cKO})$. Since previous work demonstrated that CamKII $\alpha$-cre mice are indistinguishable to wild-type littermates, we employed $A p c 2^{\mathrm{f} / \mathrm{f}}$ mice as control groups (Minichiello et al. 1999; Wirth et al. 2004). When compared to control littermates quantitative real time PCR (qPCR) analysis revealed a significant reduction of $A p c 2$ mRNA levels in the cortex and hippocampus of adult $A p c 2 c K O$ mice. The reduction of $A p c 2$ mRNA was not complete since the CamKII $\alpha$-CRE mediated deletion is specific to excitatory neurons and does not affect other cell types such as astrocytes that also express a functional APC/C (HerreroMendez et al. 2009). Consistent with the expression pattern of cre recombinase in CamKI $\alpha$-CRE mice (Minichiello et al. 1999) Apc2 expression in the cerebellum was not affected (Fig. 1C). The reduced gene-expression of $A p c 2$ in the adult forebrain resulted in reduced APC2 protein levels in the hippocampus and cortex of Apc2 cKO mice while protein levels in the cerebellum were similar among groups (Fig. 1D). In line with these data we detected a significant up-regulation of $\mathrm{CDH} 1$ protein levels in cortical and hippocampal lysates of Apc2 cKO mice (Fig. 1E). $\mathrm{CDH} 1$ is a coactivator of the APC/C that was shown to be itself an APC/C substrate (Listovsky et al. 2004). Thus, elevated CDH1 levels in Apc2 cKO mice are likely due to a failure in CDH1 degradation and are therefore indicative for impaired APC/C activity. In line with this CDH1 levels in the cerebellum were not altered (Fig. 1E).

Importantly, while deletion of $A p c 2$ during development is lethal in mice (Wirth et al. 2004), Apc2 cKO mice were indistinguishable from control littermates and showed normal home cage behavior (Fig. 2A). Brain weight was similar among Apc2 
A

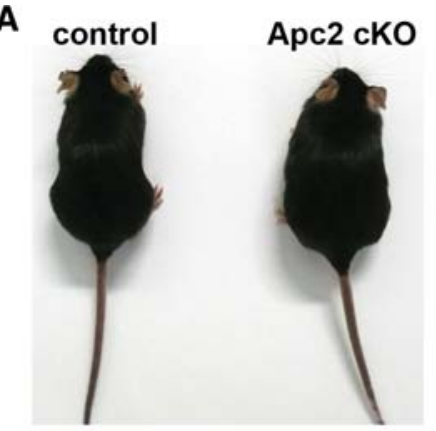

D

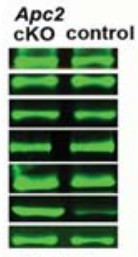

Cortex

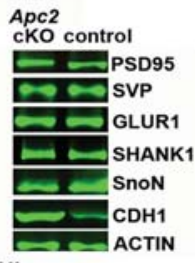

Hippocampus
B
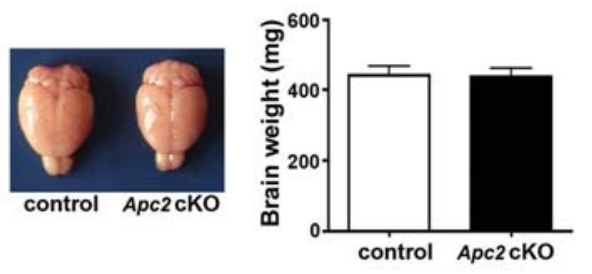

C
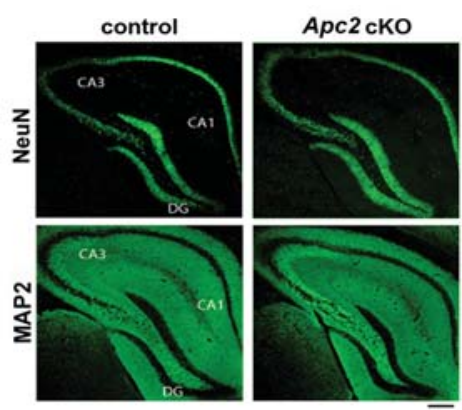

$\left.{ }^{6}\right] \quad$ CDH1 PSD95

SVP

GLUR1

SHANK1

SnoN
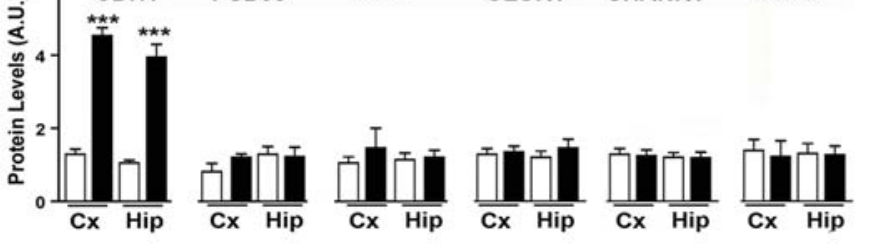

Apc2 2 KKO

Figure 2. Normal brain morphology in Apc2 cKO mice. ( $A$ ) Image of an Apc2 cKO mice and a control littermate. ( $B$ ) Representative image of a brain from an $A p c 2$ cKO and a control mouse (right panel). Quantitative analysis of brain weight (left panel). (C) Representative images showing MAP2 and NEUN immunoreactivity in Apc2 cKO and control mice. Scale bar: $100 \mu \mathrm{m}$. (D) Representative immunoblot images showing equal levels of PSD-95, GLUR1, and GLUR2 levels in Apc2 cKO and control mice (left panel). Quantification $n=4-6 /$ group (right panel). CA1, CA3, hippocampal subfields Ca1 and Ca3; DG, dentate gyrus. Error bars indicate SEM.

cKO and control mice (Fig. 2B). Immunoreactivity of microtubule-associated protein 2 (Map2) and Neuronal N (NeuN), two well-established markers for neuronal integrity (Fischer et al. 2007; Peleg et al. 2010), was similar among Apc2 cKO and control mice (Fig. 2C). As such, our data suggest that gross brain morphology and neuronal integrity is normal in $\mathrm{Apc} 2 \mathrm{cKO}$ mice. In line with this immunoblot analysis of hippocampal lysates revealed that proteins levels of markers for neuronal integrity such as postsynaptic density-95 (PSD95), synaptophysin (SVP), or Glutamate receptor 1 (GLUR1) or SH3 and multiple ankyrin repeat domains protein 1 (SHANK1) were similar among groups (Fig. 2D). While we could confirm significant up-regulation of $\mathrm{CDH}-1$ levels in $A p c 2$ cKO mice, we did not observe differences in the levels of the APC/C target SnoN (Fig. 2D). In summary, this data indicates that loss of APC2 from excitatory neurons of the adult forebrain of mice does not cause an overt phenotype.

\section{Basal anxiety, motor-coordination, explorative and} depressive-like behavior is normal in Apc2 cKO mice

Next, we exposed male $A p c 2 \mathrm{cKO}$ and control mice to the open field paradigm, a simple test for basal anxiety, explorative behavior, and locomotion. We did not observe a significant difference in the distance traveled during the test period among groups, indicating that explorative and locomotor behavior in a novel context is normal in Apc2 cKO mice (Fig. 3A,B). No difference was observed when we specifically analyzed the time spent in the center vs. the border of the open field arena, a common measure of anxiety, suggesting that basal anxiety was similar among groups (Fig. 3C). To investigate this in greater detail we subjected a group of male $A p c 2 \mathrm{cKO}$ and control mice to the elevated plus maze test, another paradigm that allows the assessment of anxiety levels in rodents. Both groups spent similar times in the closed vs. open arms of the maze (Fig. 3D). In conclusion this data demonstrates that loss of APC2 in the forebrain of mice does not affect explorative behavior and basal anxiety. In addition we did not observe significant group differences when male Apc2 $\mathrm{cKO}$ and control mice were exposed to the porsolt forced swim test (Fig. 3E) and well-established paradigm to analyze depressive like behavior. We also examined the performance of male Apc2 cKO mice and control littermates in the accelerated Rotarod test, a motor coordination task, but did not observe significant differences among groups (Fig. 3F). Similarly, basal anxiety, explorative and depressive-like behavior as well as motor coordination were similar amongst female $A p c 2 \mathrm{cKO}$ and control mice (data not shown).

\section{Apc2 cKO mice display impaired spatial memory}

To test learning and memory function $A p c 2 \mathrm{cKO}(n=16)$ and control mice $(n=17)$ were subjected to the Morris water maze test, a hippocampal-dependent assay for spatial memory performance. All groups improved in their ability to find the hidden platform. There was no significant gender effect in control $(P=0.9223$, $F=0.3969)$ or $A p c 2$ cKO mice $(P=0.1677, F=1.462)$. However, $A p c 2$ cKO displayed significantly impaired escape latency on training days 3-4 and 6-10 when compared to the control group. In line with this, repeated measures ANOVA showed that the escape latency throughout the 10 training days, an indicator of spatial learning, was significantly impaired $(P<0.0001, F=55.01$ vs. control) in $A p c 2 \mathrm{cKO}$ mice when compared to the corresponding control group (Fig. 4A). Since groups did not differ in finding a visible platform (Fig. 4A) or the swimming speed during the training (Fig. 4B) our data suggests that $A p c 2$ CKO mice are impaired in spatial learning ability. Consistently, when compared to the control group, Apc2 cKO spent significantly less time in the target quadrant during a probe test performed after $10 \mathrm{~d}$ of training (Fig. 4C). Similarly, the number of platform crossings was reduced in Apc2 cKO mice (Fig. 4D), which is indicative of impaired spatial 
A

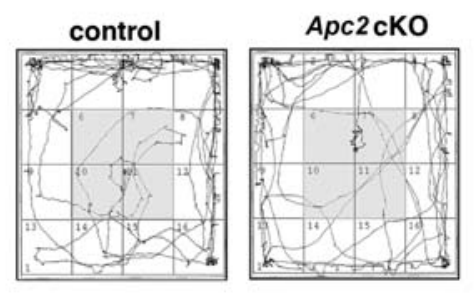

C

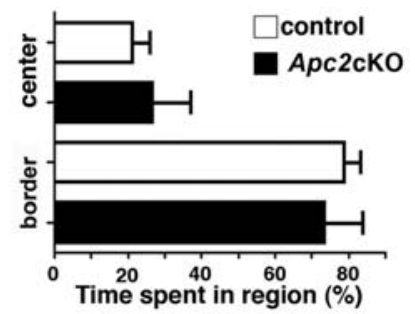

E

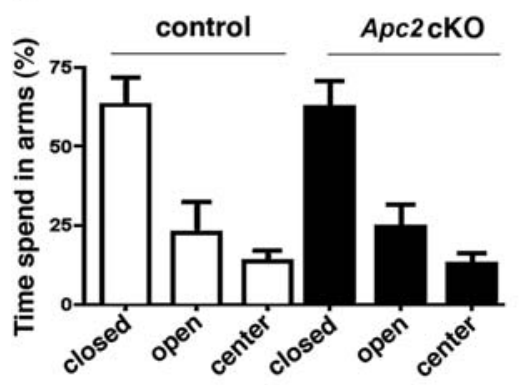

B

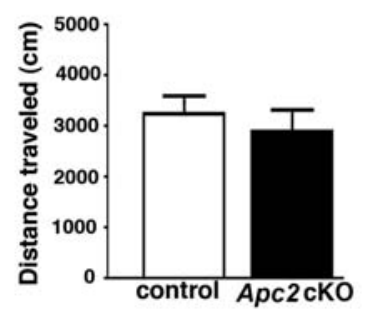

D

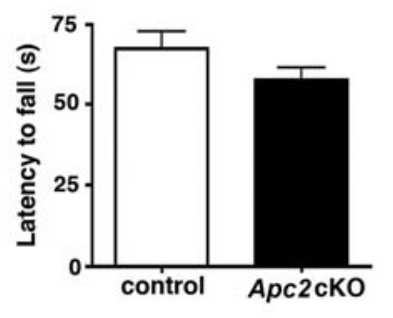

$\mathbf{F}$

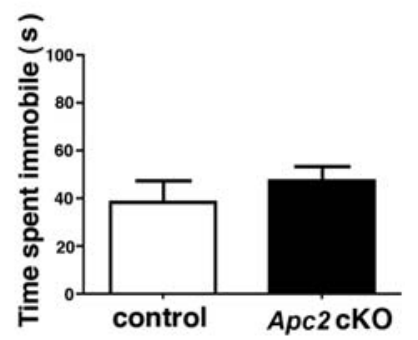

Figure 3. Basal anxiety, explorative and depressive-like behavior, and motor coordination in Apc2 cKO mice. (A) Representative image showing the explorative behavior of Apc2 cKO and control mice in an open field. (B) Distance traveled during $5 \mathrm{~min}$ exposure to an open field. (C) Time spent in the center vs. the corner regions of the open field during a 5 min test session. (D) Apc2 cKO and control mice were subjected to the rotarod test. No significant difference was observed among groups. $(E)$ When exposed to the elevated plus maze test Apc2 cKO and control mice spent similar times in the center, the closed or the open arms. $(F) A p c 2$ cKO and control mice were subjected to the Porsolt forced swim test. The time spent immobile was similar among groups. $n=8-12 /$ group. Error bars indicate SEM.

memory. When exposed to a reversal-learning paradigm in which the platform was moved to a new location, Apc2 cKO also performed significantly worse than the corresponding control group (Fig. 4E). In conclusion this data shows that spatial memory performance is impaired in $A p c 2$ cKO mice.

\section{Apc2 cKO mice exhibit severely impaired extinction of fear memories}

To further assess the role of $A p c 2$ in memory function mice were subjected to the Pavlovian fear conditioning paradigm, a standardized test to measure associative learning in rodents (Fischer et al. 2007). Male Apc2 cKO and control mice were subjected to contextual fear conditioning. The activity during the training and the response to the electric footshock was similar among groups (Fig. 5A). When freezing behavior was analyzed in a memory test performed $24 \mathrm{~h}$ later no difference was observed among groups (Fig. 5B). Similar results were obtained for tone-dependent fear conditioning (Fig. 5C) or when mice were subjected to a weaker training protocol (Supplemental Fig. 1). We conducted the same experiments in female $A p c 2$ cKO and control mice. While explorative behavior during the training and the response to the footshock was similar among groups (Fig. 5D), Apc2 cKO mice displayed significantly more freezing behavior during a contextual memory test performed $24 \mathrm{~h}$ after the training (Fig. 5E). Similar data were observed during tone-dependent fear conditioning (Fig. 5F). Interestingly, this data indicates that the consolidation of fear memories is normal in male and even enhanced in female mice that lack APC2 in the adult forebrain.

A strong association between a fear-eliciting and an otherwise neutral stimulus, such as the electric foot shock and the novel context in the fear conditioning paradigm, is often the basis for anxiety diseases such as phobia or posttraumatic stress disorder (Myers and Davis 2007; Sananbenesi et al. 2007). Treatment of such disorders involves repeated exposure to the frightening stimulus in the absence of the aversive event, which gradually leads to the reduction of the fear memory response, a process named fear extinction. Fear extinction is often impaired in patients suffering from anxiety diseases. While the mechanisms underlying fear extinction are not well understood, a recent study implicated proteasome activity with the extinction of fear memories (Lee et al. 2008). To this end we set out experiments to analyze whether APC2 plays a role in fear extinction. Male Apc $2 \mathrm{cKO}$ mice and a corresponding control group were subjected to contextual fear conditioning and subsequently exposed to extinction training, consisting of reexposure to the conditioning context on consecutive days in the absence of the footshock (Fischer et al. 2004; Sananbenesi et al. 2007; Tronson et al. 2009). While freezing behavior declined throughout extinction training in the control group, Apc2 cKO displayed high freezing on all extinction trials (Fig. 6A). Similar data was obtained in female mice (Fig. 6B). However, as shown before (see Fig. 5E) female $A p c 2$ cKO mice already displayed elevated freezing behavior on extinction trial 1 (E1), which may compromise the observed effect. Nevertheless, impaired fear extinction in female Apc2 cKO mice was still obvious when freezing levels on E1 were normalized to allow a direct comparison among groups (Fig. 6C). This data demonstrates that fear extinction is severely impaired in male and female $A p c 2 \mathrm{cKO}$ mice.

\section{Loss of Apc2 from the adult forebrain does not influence disease progression in a mouse model for amyloid pathology}

Recent in vitro studies implicated deregulated APC/C function with neuronal cell death and it has been suggested that loss of APC/C activity might play a role in the pathogenesis of Alzheimer's disease (Almeida et al. 2005; Maestre et al. 2008). To 

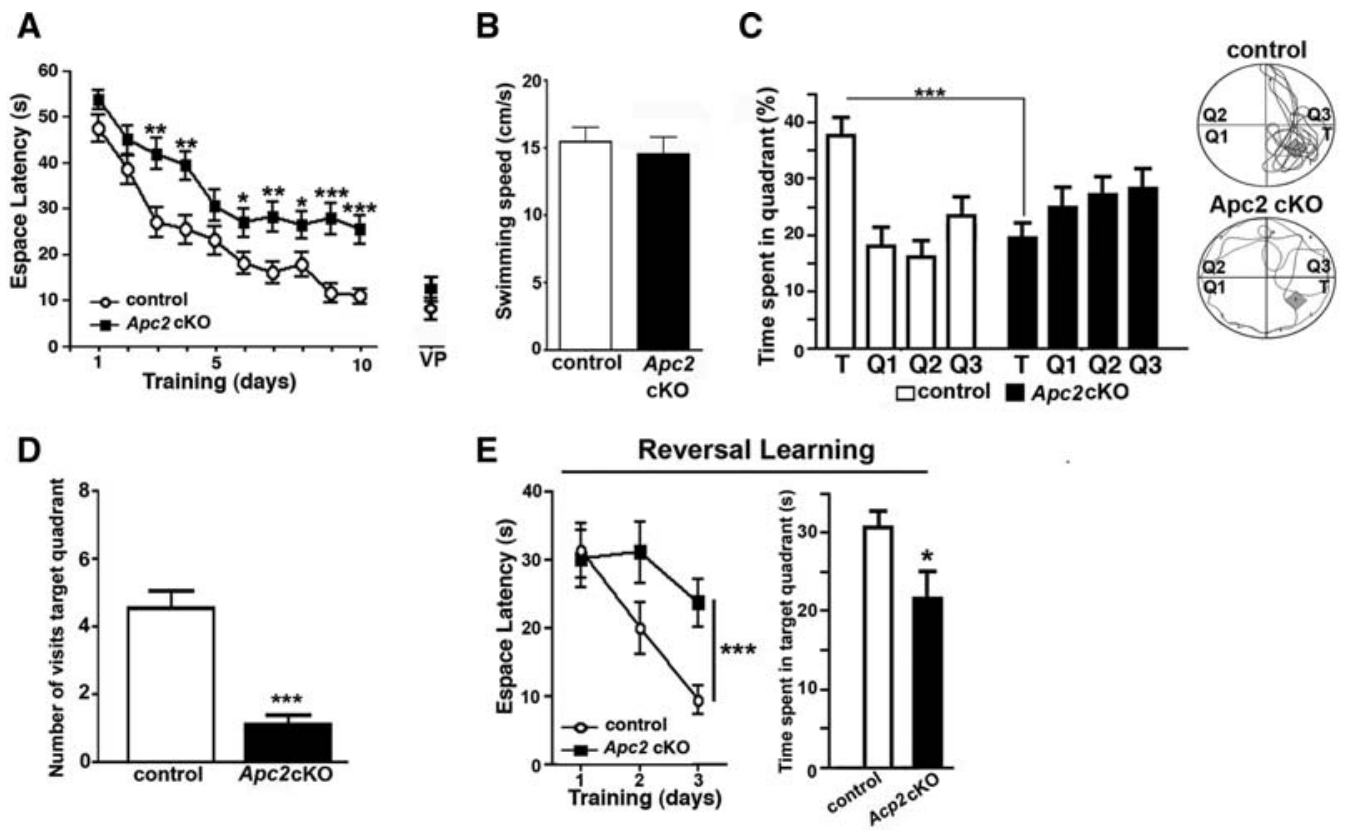

Figure 4. Impaired spatial memory formation in $A p c 2 \mathrm{cKO}$ mice. (A) When compared to the control group $(n=16)$, the escape latency throughout the training was significantly impaired in $\operatorname{Apc} 2 \mathrm{cKO}$ mice $(n=17 ; P<0.0001, F=55.01$ vs. control). Groups did not significantly differ in finding a visible platform (VP). (B) Swimming speed during the days of training was similar among groups. (C) After $10 \mathrm{~d}$ of training Apc2 cKO and control mice were subjected to a probe trial. The time spent in the target quadrant was significantly impaired in Apc2 cKO mice when compared to the control group $(P<0.0001)$. The right panel shows representative swim path of $A p c 2$ cKO and control mice during the probe test. $(D)$ During the probe test the number of visits to the target quadrant was significantly reduced in $A p c 2 \mathrm{cKO}$ mice $(P<0.0001$ vs. control). (E) Reversal learning was impaired in Apc2 cKO mice when compared to control mice. (Left panel) Escape latency during the reversal training $(P<0.05 ; F=5.699$ vs. control); (right panel) time spent in the new target quadrant during the probe test $(P<0.05$ vs. control). T, target quadrant, Q1, quadrant 1; Q2, quadrant 2; Q3, quadrant 3; Q4, quadrant 4. Error bars indicate SEM.

further test this possibility we crossed Apc2 cKO mice with APP/ PS1 mice, a mouse model for severe amyloid pathology (Radde et al. 2006). We analyzed learning behavior in 6-mo-old female Apc2 cKO/APP/PS1 mice, because that age marks the onset of memory disturbances and thus should allow us to observe either an enhancement or an impairment of learning abilities (Fig. 7A). When trained in the Morris water maze test APP/PS1 and Apc2 cKO/APP/PS1 mice showed no significant difference with respect to the escape latency (Fig. 7B,C). Similarly, when a probe test was performed after $10 \mathrm{~d}$ of training, the performance of $A p c 2 \mathrm{cKO}$ and $A p c 2 \mathrm{cKO}$ APP/PS1 mice was similar and both groups showed a nonsignificant preference for the target quadrant (Fig. 7D). When trained in the contextual fear conditioning paradigm no significant difference was observed among groups (Fig. 7E). This data suggests that loss of APC2 does not affect memory function in APP/PS1 mice APP/PS1 mice are characterized by a very aggressive amyloid pathology and $A ß$ plaques are already observed in 2-mo-old mice (Radde et al. 2006). When we analyzed the Aß-plaque load in the hippocampal region of APP/PS1 and Apc2 cKO/APP/PS1 mice, both groups exhibited similar amyloid pathology (Fig. 7F). In line with this data, no difference in NeuN or MAP2 levels was observed when analyzed in the hippocampal region by quantitative immunohistochemistry (Fig. 7G). This data suggests that in our experimental setting loss of Apc2 from the adult forebrain does not affect hippocampus-dependent learning behavior and amyloid-plaque load in APP/PS1 mice.

\section{Discussion}

In this work we investigated the role of APC/C activity during learning and memory by specifically deleting the essential
APC/C subunit APC2 from postmitotic excitatory neurons of the adult forebrain in mice. To this end we crossed $A p c 2^{\mathrm{fl} / \mathrm{fl}}$ mice with transgenic animals expressing cre-recombinase under control of the CamKII $\alpha$ promoter. As expected, we observed a significant reduction of $A p c 2$ mRNA levels in the hippocampus and cortex of $A p c 2$ cKO mice whereas Apc2 mRNA levels in the cerebellum were not affected. The reduction of $A p c 2$ mRNA in the cortex and hippocampus was however not $100 \%$ since the genetic manipulation eliminates $A p c 2$ expression only in excitatory neurons, whereas glial cells, interneurons and other neural types maintain normal expression (Herrero-Mendez et al. 2009). Similarly, we observed a significant, but not complete reduction of APC2 protein in hippocampus and cortex of Apc2 cKO mice. In line with this data, we detected a significant up-regulation of $\mathrm{CDH} 1$ protein levels in hippocampal and cortical, but not in cerebellar lysates. $\mathrm{CDH} 1$ associates with $\mathrm{APC} / \mathrm{C}$ as an activator but at the same time is targeted for degradation in an APC/C dependent manner (Peters 2006). Therefore, elevated CDH1 protein levels are indicative of reduced $\mathrm{APC} / \mathrm{C}$ activity in $\mathrm{Apc} 2 \mathrm{cKO}$ mice. Loss of APC2 activity during development is lethal in mice (Wirth et al. 2004). Moreover, deletion of Apc2 from hepatocytes leads to an unscheduled entry into cell cycle causing a severe liver failure (Wirth et al. 2004). In contrast, when we postnatally deleted Apc2 from principal forebrain neurons mice were viable and did not show any overt phenotype. We also did not observe brain atrophy or gross changes in neuronal integrity suggesting that APC2 is not critical for neuronal survival in adult mice. In addition explorative behavior in a novel context and motor coordination was indistinguishable in Apc2 cKO and control mice. Apc2 cKO mice also exhibited normal basal anxiety and no change in depressive-like behavior. However, when tested in the Morris water maze paradigm, Apc2 cKO displayed impaired spatial 
A

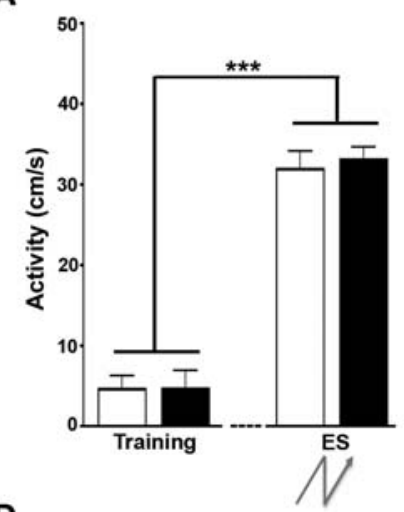

D

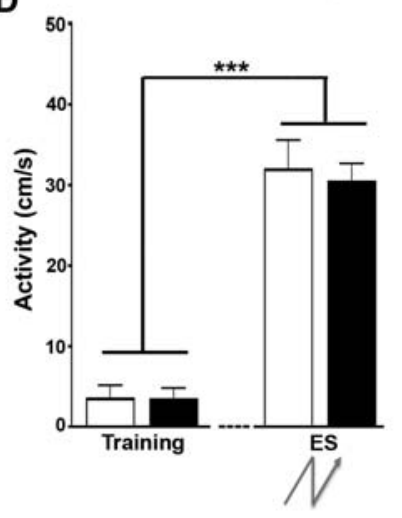

B

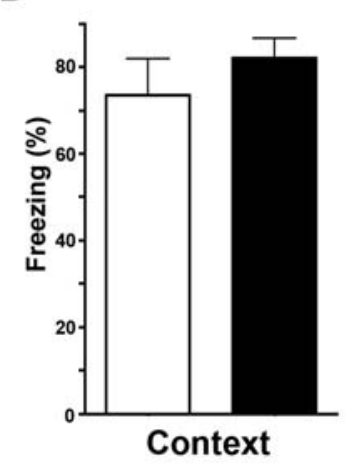

E

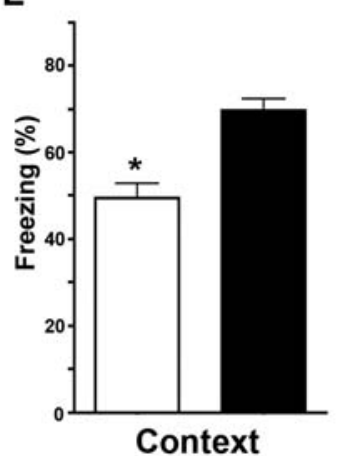

Context

$\square$ control $\square$ Apc2 cKO

Figure 5. Acquisition of fear memories in $A p c 2$ cKO mice. ( $A$ ) The activity in response to the footshock increased to a similar degree in male $A p C 2 \mathrm{CKO}$ and control animals $(P<0.0001$ training vs. ES). (B) Freezing behavior in the contextual memory test was similar in male $A p c 2$ CKO mice and control littermates. (C) Freezing behavior in the cued memory test was similar in male Apc2 cKO mice and control littermates. $(D)$ The activity in response to the footshock increased to a similar degree in female $A p c 2$ cKO and control animals $(P<0.0001$ training vs. ES). (E) Freezing behavior in the contextual memory test was significantly higher in female Apc2 cKO mice when compared to control littermates $(P<0.05)$. $(F)$ Freezing behavior in the cued memory test was significantly higher in female Apc2 CKO mice when compared to control littermates $(P<0.05) . n=9 /$ group; ES, electric footshock. Error bars indicate SEM.

memory performance. The water maze test is hippocampusdependent suggesting that hippocampal APC2 is required for the consolidation of spatial memories. This data is in line with previous findings that reported impaired spatial or associative learning in mice that were injected intrahippocampally with a protein degradation inhibitor (Lopez-Salon et al. 2001; Artinian et al. 2008). Impaired hippocampus dependent memory formation was also observed in mice that exhibited reduced CDH1 levels, a critical APC/C coactivator. In this study complete Cdh1 knock out mice were used and while homozygousity was lethal, heterozygous animals were viable and displayed impaired hippocampus-dependent memory formation (Li et al. 2008). It is therefore interesting that hippocampal and cortical CDH1 levels were elevated in our $A p c 2 \mathrm{cKO}$ mice. However, this is most likely due to impaired APC/C function, which normally targets CDH1 for degradation (Listovsky et al. 2004). As such the elevated Cdh1 levels in Apc2 cKO mice reflect a failure in Cdh1 degradation. Importantly, hippocampal levels of other potential of known APC/C targets such as SnoN or SHANK1, which was shown to be regulated via ubiquitination (Lee et al. 2008), were not altered in Apc2 cKO mice. This data indicates that different APC/C substrates play a role in APC/C mediated memory function. Moreover, the APC/C can interact with different E2 ligases
C

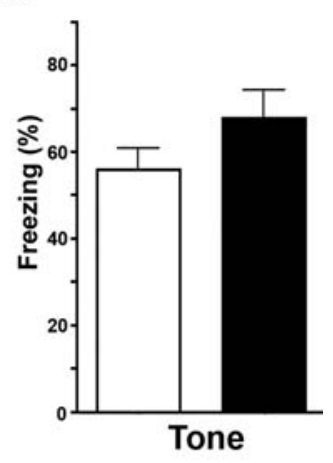

$\mathbf{F}$

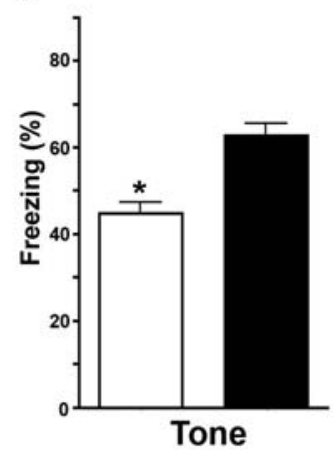

and it is possible that such interactions are rather dynamic and induced via specific environmental stimuli. Thus, the possibility remains that known and unknown APC/C substrates are deregulated in $A p c 2 \mathrm{cKO}$ mice only in response to specific environmental stimuli.

Interestingly Pavlovian fear conditioning that is commonly used to measure associative fear memories in rodents was not affected in male $A p c 2 \mathrm{cKO}$ mice and even enhanced in female $A p c 2$ cKO mice. Although spatial memory in the water maze paradigm and contextual fear conditioning are hippocampus dependent, the fear conditioning paradigm specifically tests for the acquisition of fear memories. It is therefore important to reiterate that excessive fear memory is often the basis for neuropsychiatric disorders such as phobia or post-traumatic stress disorder (PTSD), which severely affect the life of patients and are an increasing burden to our societies (Sotres-Bayon et al. 2006; Myers and Davis 2007; Bremner et al. 2008; Fischer and Tsai 2008; Hartley and Phelps 2010). Therapeutic strategies to inhibit excess fear are therefore central to the treatment of anxiety and mood disorders. Fear inhibition can be achieved by repeated reexposure to the fear-eliciting stimulus in the absence of any aversive event. This normally leads to a gradual reduction of fear, a process named fear extinction. Interestingly, we observed that male and female $A p c 2$ cKO mice exhibited severely impaired fear extinction. As such, the aversive freezing behavior did not decline throughout our extinction training protocol and was still significantly higher than in control mice when tested $28 \mathrm{~d}$ after the last extinction trial. This data indicates that APC2 function is of particular importance for the extinction of fear memories and indicates that the APC/C may play a role in the pathogenesis of neuropsychiatric diseases. In line with this view a recent study demonstrated that proteasomal protein degradation in the hippocampus is required for fear extinction in mice (Lee et al. 2008). Moreover, while the partial NMDA receptor agonist D-cyloserine (DCS) facilitates fear extinction in rodents and also exhibits beneficial effects in patients suffering from phobia (Ressler et al. 2004; Davis et al. 2006) the effect of DCS injection was blocked when coadministered with proteasome inhibitors (Mao et al. 2008). While proteasome inhibitors are rather unselective our data strongly suggest that the APC/C is one of the players mediating the effect of proteasomal protein degradation during the extinction of fear memories. While the precise mechanisms affected by APC/C during fear extinction remain to be elucidated it is interesting to note that cell culture studies provided substantial evidence that proteasomal protein degradation is critical for synaptic plasticity, especially the formation and dynamics of dendritic spines (Mabb and Ehlers 2010). As such, morphological or functional reorganization of particular hippocampal synapses could be a likely downstream mechanism of APC/C activity during fear extinction. 
A

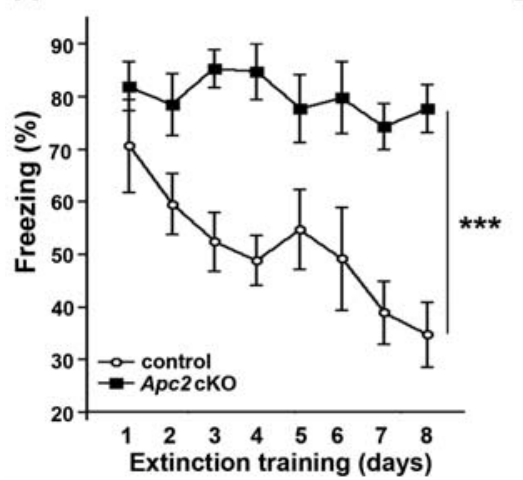

B

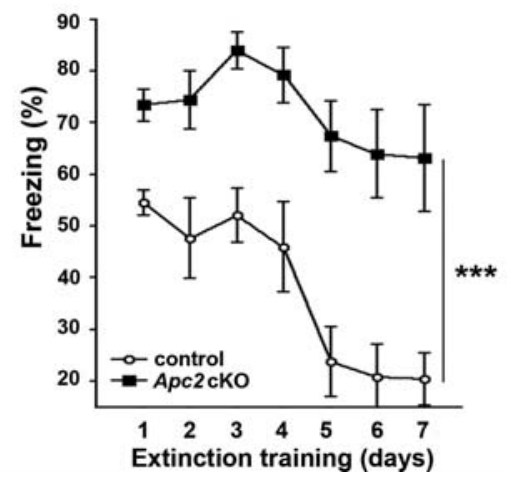

C

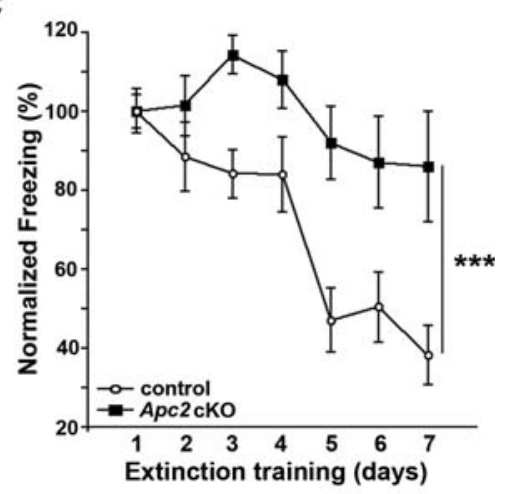

Figure 6. Impaired extinction of fear memories in $A p c 2 \mathrm{cKO}$ mice. (A) The extinction of contextual fear memories was significantly impaired in male Apc2 cKO mice when compared to a control group $(* * * P<0.0001$, repeated measurements ANOVA $P<0.0001, F=19.84)$. (B) The extinction of contextual fear memories was significantly impaired in female Apc2 CKO mice when compared to a control group ( $* * *^{*}<0.0001$, repeated-measurements ANOVA $P<0.0001, F=5.85$ ). (C) The data shown in $B$ was normalized so that freezing on extinction trial 1 was similar among groups. $n=9 /$ group. Error bars indicate SEM.

Dysfunction of proteosomal degradation has been implicated with the pathogenesis of various neurodegenerative diseases including Alzheimer's disease (Oddo 2008). Recent evidence specifically points to a role of deregulated APC/C function during AD-like neurodegeneration. For example, RNA interference mediated down-regulation of $\mathrm{CDH} 1$ in cultured hippocampal neurons induced apoptotic cell death, most likely due to cell cycle reentry (Almeida et al. 2005). Moreover, CDH1 is a target of the cyclinedependent kinase 5 (Cdk5) which, when activated by the p25 protein, has been implicated with neurodegeneration and learning impairments (Fischer et al. 2005, 2007; Maestre et al. 2008). To test a function of APC/C in the pathogenesis of Alzheimer's disease we crossed Apc2 cKO mice to APP/PS1 mice, which develop severe amyloid pathology and memory deficits (Radde et al. 2006). However, in our experimental settings spatial and associative memory function was indistinguishable between $A p c 2 \mathrm{cKO}$ and $A p c 2 \mathrm{cKO} / \mathrm{APP} / \mathrm{PS} 1$ mice. In addition, amyloid pathology, as measured as Aß-plaque load, was indistinguishable among groups. This data indicates that APC/C activity has no influence on the amyloid pathology and the associated memory impairment in the employed mouse model. The fact that loss of APC2 does not aggravate memory deficits in APP/PS1 mice may also suggest that the mechanisms by which amyloid-ß-pathology and loss of APC2 affect memory function might share similar mechanism of action. However, amyloid pathology is only one pathological hallmark of Alzheimer's disease and it is important to note that neuronal cell death is usually not detectable at significant levels in mouse models for amyloid pathology. As such, the effect of APC/C activity on Alzheimer's related neuronal cell death or other pathological features such as Tau pathology remains to be investigated in future studies.

In conclusion, our study shows that APC/C activity in principle forebrain neurons is essential for cognitive function. At least in our experimental settings APC/C seems to have only limited impact on neuronal survival in adulthood and Alzheimer's disease related amyloid-pathology. Interestingly, our data indicates that loss of APC2 recapitulate some, but not all effects on learning behavior that has been observed with pharmacological inhibition proteasome activity. As such, while the acquisition of fear memories was not affected or even enhanced in female $A p c 2 \mathrm{cKO}$ mice, $\mathrm{APC} / \mathrm{C}$ activity seems to be of particular importance for the extinction of fear memories. Thus, our data indicate that APC/C activity in the adult forebrain may play a role in the pathogenesis of anxiety diseases.

\section{Materials and Methods}

\section{Animals}

Mice were housed under standard conditions with free access to food and water. All experiments were carried out in accordance with the animal protection law and were approved by the District Government of Germany. The genetic background of all mice was $\mathrm{C} 57 \mathrm{Bl} / 6 \mathrm{~J}$. The generation of Apc $2^{\mathrm{f} / \mathrm{f}}$, CamKII $\alpha$-cre, and APP/PS1 mice has been described previously (Minichiello et al. 1999; Wirth et al. 2004; Radde et al. 2006).

\section{Behavioral analysis}

Behavior testing was performed as described previously (Fischer et al. 2004; Park et al. 2005; Peleg et al. 2010). In brief, mice were single housed and habituated to the testing room at least 3 $\mathrm{d}$ before behavior experiments. For the open field test mice were placed in the center of a plastic arena (length $1 \mathrm{~m}$; width $1 \mathrm{~m}$; side walls $20 \mathrm{~cm}$ height) for $5 \mathrm{~min}$. The explorative behavior was recorded by a camera and analyzed using the VideoMot2 software (TSE Systems). For elevated plus maze analysis mice were placed in the center region of the elevated maze facing the open arm. The behavior was recorded for 5 min using the VideoMot2 system (TSE systems). The Porsolt forced swim test was conducted by exposing mice for $5 \mathrm{~min}$ to a container (diameter of $20 \mathrm{~cm}$ ) that was filled with water. An observer that was blind to the genotype measured immobility. Fear conditioning training was performed using the TSE fear conditioning system. The procedure consisted of exposing mice to the conditioning context $(3 \mathrm{~min})$ followed by a single electric footshock $(0.7 \mathrm{~mA}$, constant current, $2 \mathrm{sec})$. Afterward, mice were left in the conditioning box for $15 \mathrm{sec}$ before being returned to their home cage. Freezing was analyzed $24 \mathrm{~h}$ later during reexposure to the conditioning context. To control for potential ceiling effects in male $A p c 2 \mathrm{cKO}$ mice an additional fear conditioning test was performed using lower shock intensity of $0.5 \mathrm{~mA}$ (constant current, $2 \mathrm{sec}$ ). For cued-dependent fear conditioning, a tone $(10 \mathrm{kHz}, 75 \mathrm{db})$ was presented for $30 \mathrm{sec}$ prior to the footshock and tone-dependent memory was tested by placing the mice into a novel context for $1 \mathrm{~min}$ followed by $3 \mathrm{~min}$ exposure to the conditioning tone. For fear extinction, mice were subjected to extinction trials (E) on consecutive days. Unless otherwise stated, contextual and cued-conditioning was combined as one training session. In this case mice were first tested for contextual fear memory after $24 \mathrm{~h}$ and then tested for cued conditioning another $24 \mathrm{~h}$ later. Each extinction trial consisted of $3 \mathrm{~min}$ reexposure to the conditioning context. The water maze training was performed in a circular tank (diameter $1.2 \mathrm{~m}$ ) filled with opaque water. A platform $(11 \times 11 \mathrm{~cm})$ was submerged 
A

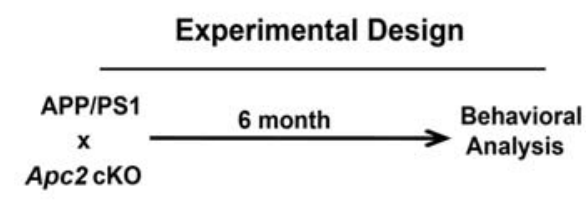

C

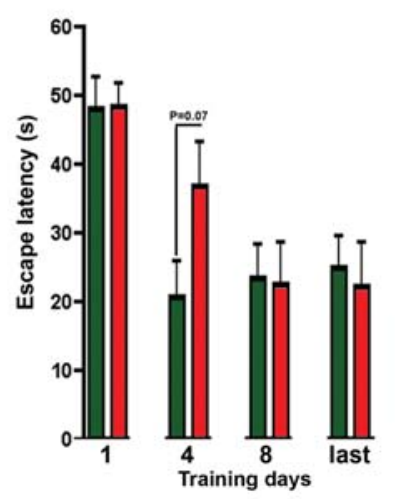

E

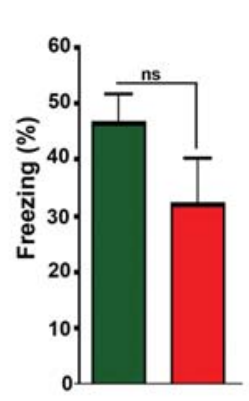

B

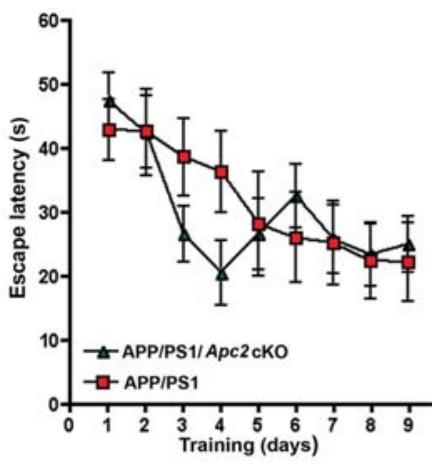

D
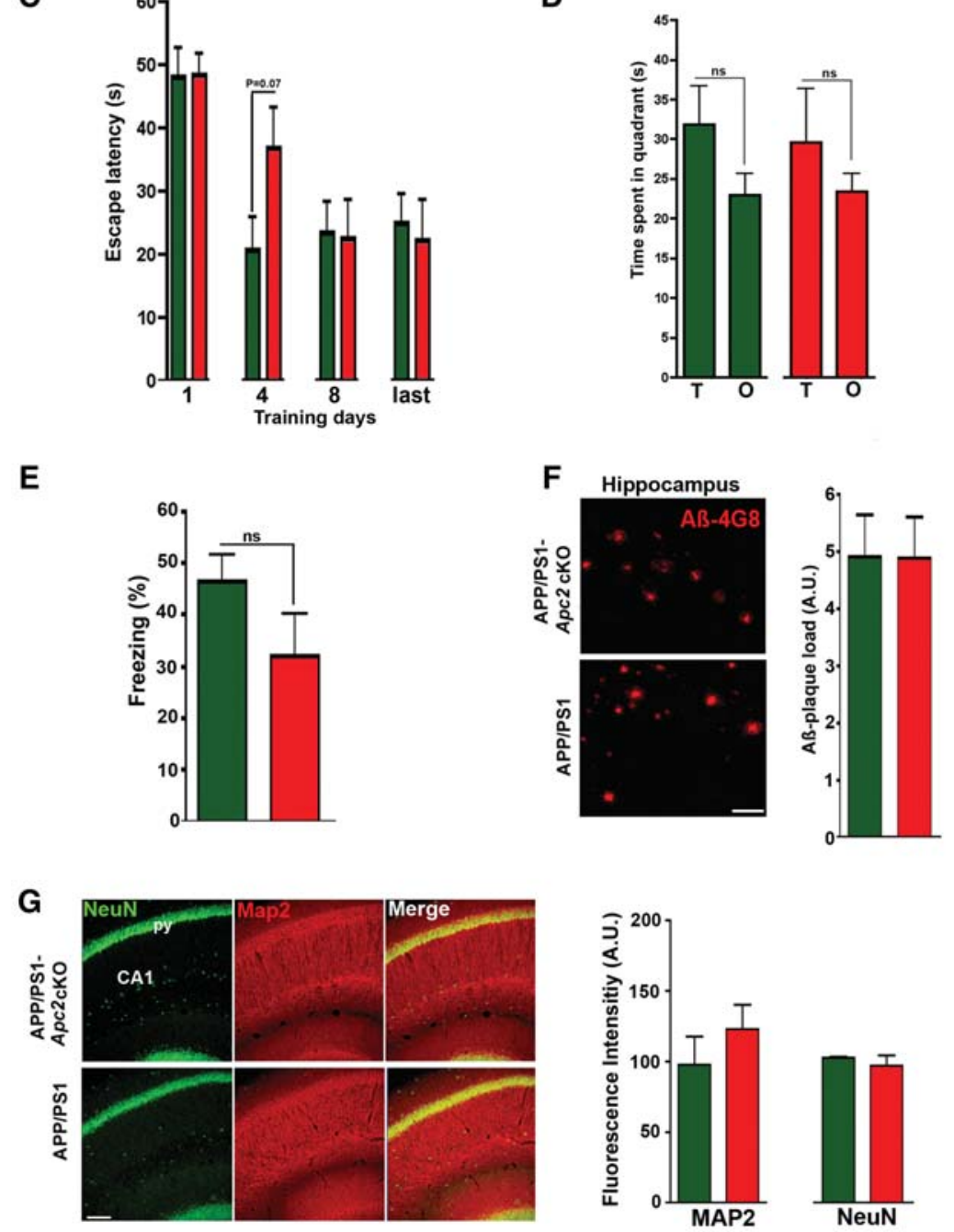

APP/PS1-Apc2cKO

APP/PS1

Figure 7. Loss of $A p c 2$ in principal forebrain neurons does not affect pathology in APP/PS1 mice. (A) Experimental design. $(B)$ The escape latency during the water maze training was similar among Apc2 cKO and $A p c 2$ cKO/APP/PS1 mice. (C) The escape latency was analyzed on individual training days. No significant changes were observed among groups. $(D)$ Time spent in the target quadrant was similar among groups. Both groups showed a nonsignificant preference for the target quadrant. $(E)$ Contextual freezing behavior was similar among groups. (F) Aß-plaque load was did not significantly differ among groups. (Left panel) Representative images showing Aß-plaque load in the hippocampus; (right panel) quantification. (G) Neuronal integrity as measured by NeuN and MAP-2 staining was not different among groups. (Left panel) Representative images; (right panel) quantification. $n=10 /$ group for behavior experiments, $n=3 /$ group for immunohistochemistry; $\mathrm{T}$, target quadrant; $\mathrm{O}$, other quadrants. Error bars indicate SEM. below the water's surface in the center of the target quadrant. The swimming path of the mice was recorded by a video camera and analyzed by the Videomot 2 software (TSE). For each training session, the mice were placed into the maze subsequently from four random points of the tank and were allowed to search for the platform for $60 \mathrm{sec}$. If the mice did not find the platform within $60 \mathrm{sec}$, they were gently guided to it. Mice were allowed to remain on the platform for $15 \mathrm{sec}$. Mice were subjected to one memory test (probe trial) $24 \mathrm{~h}$ after the last training session. During the probe test the platform was removed from the tank and the mice were allowed to swim in the maze for $60 \mathrm{sec}$. For the visual Morris water maze test, mice were habituated to the pool for two consecutive days. During the visual probe test the platform was placed in the pool and made visible by an elevated cube. For reversal learning the platform was transferred to a new quadrant. The Rotarod test was conducted using the Rota Rod V4.02 System from TSE Systems. Mice were first habituated to the procedure and then subjected to an accelerated paradigm for $4 \mathrm{~min}$ on two consecutive days (10-40 rpm).

\section{Immunoblot analysis and immunohistochemistry}

Brain tissue was homogenized in TX buffer $(50 \mathrm{mM}$ Tris, $150 \mathrm{mM} \mathrm{NaCl}, 2 \mathrm{~m}$ EDTA, $1 \%$ Triton- $X$, and protease inhibitors) incubated for $15 \mathrm{~min}$ at $4{ }^{\circ} \mathrm{C}$ and centrifuged for $10 \mathrm{~min}(10,000 \mathrm{rpm})$. The supernatant was used for immunoblotting. Immunoblots were performed using fluorescent secondary antibodies and data were quantified using an Odyssey Imager (Licor). Antibodies were diluted either in $0.5 \%$ milk PBT or $0.5 \%$ milk TBT, respectively. The following antibodies were commercially purchased and used in the mentioned concentrations: $\beta$-Actin (Ac-15) 1:1000, Santa Cruz Biotechnology; GAPDH, 1: 5000, Chemicon; GluR1 1:1000, Synaptic Systems; Synaptophysin (Syp38) 1:1000, Sigma-Aldrich, Map2, 1:1000; Synaptic Systems, NeuN 1:1000, Chemicon. The antibody against APC2 was a gift from J.M. Peters. APC2 hybridoma cell supernatant was used in a 1:200 dilution. The antibody used to detect Cdh1 was a gift by J. Gannon. In this case, $66 \mu \mathrm{l}$ of the hybridoma cell supernatant were dissolved in $2 \%$ BSA in TBT. Immunostaining was performed as described previously (Fischer et al. 2007) and analyzed using a Leica SP2 confocal microscope. Microtubule associated protein 2 (Map2) antibody was from SynapticSystems $(1: 200)$ and $\beta$-amyloid, 17-24 (4G8) antibody was from Convance (1:1000).

\section{Quantitative real time PCR (qPCR)}

qPCR was performed using a Roche 480 light cycler. cDNA was generated using 
the Transcriptor High Fidelity cDNA Synthesis Kit (Roche) and qPCR for individual genes were performed using the Roche Universal probe library (UPL). Data was normalized to the housekeeping gene hypoxanthine phosphoribosyltransferase (Hprt).

\section{Statistical analysis}

The data was analyzed by unpaired student's $t$-test and one-way or two-way ANOVA (ANalyis Of VAriance) when appropriate. Errors are displayed as standard error of the mean (SEM).

\section{Acknowledgments}

We thank Dr. Judith Stegmüller for reading the manuscript and helpful comments. We would also like to thank E. Fuchs for insightful comments on the project. We thank J.M. Peters for the APC2 antibody and J. Gannon for the Cdh1 antibody. This work was supported by the following grants to A.F.: the EURYI award and the ERA-Net Neuron Project Epitherapy. F.S. and S.B. are supported by the research grant SA/1050/2-1 of the German Research Foundation (DFG). T.K. was supported by a PhD fellowship from Boehringer Ingelheim Fonds.

\section{References}

Almeida A, Bolaños JP, Moreno S. 2005. Cdh1/Hct1-APC is essential for the survival of postmitotic neurons. J Neurosci 26: 8115-8121.

Artinian J, McGauran AM, De Jaeger X, Mouledous L, Frances B, Roullet P. 2008. Protein degradation, as with protein synthesis, is required during not only long-term spatial memory consolidation but also reconsolidation. Eur J Neurosci 27: 3009-3019.

Barco A, Lopez de Armentia M, Alarcon JM. 2008. Synapse-specific stabilization of plasticity processes: The synaptic tagging and capture hypothesis revisited 10 years later. Neurosci Biobehav Rev 32: $831-851$.

Bremner JD, Elzinga B, Schmahl C, Vermetten E. 2008. Structural and functional plasticity of the human brain in post-traumatic stress disorder. Prog Brain Res 167: 171-186.

Ciechanover A. 2005. Proteolysis: From the lysosome to ubiquitin and the proteasome. Nat Rev Mol Cell Biol 6: 79-87.

Davis M, Ressler K, Rothbaum BO, Richardson R. 2006. Effects of D-cycloserine on extinction: Translation from preclinical to clinical work. Biol Psychiatry 60: 369-375.

Fischer A, Tsai LH. 2008. Counteracting molecular pathways regulating the reduction of fear: Implications for the treatment of anxiety diseases. In Post traumatic stress disorders: basic science and clinical practice (ed. PJ Shiromani, et al.), pp. 79-104. Humana Press, New York, NY.

Fischer A, Sananbenesi F, Schrick C, Spiess J, Radulovic J. 2004. Distinct roles of hippocampal de novo protein synthesis and actin rearrangement in extinction of contextual fear. J Neurosci 24: $1962-1966$.

Fischer A, Sananbenesi F, Pang PT, Lu B, Tsai LH. 2005. Opposing roles of transient and prolonged expression of p25 in synaptic plasticity and hippocampus-dependent memory. Neuron 48: 825-838.

Fischer A, Sananbenesi F, Wang X, Dobbin M, Tsai LH. 2007. Recovery of learning and memory after neuronal loss is associated with chromatin remodeling. Nature 447: 178-182.

Gieffers C, Peters BH, Kramer ER, Dotti CG, Peters JM. 1999. Expression of the CDH1-associated form of the anaphase-promoting complex in postmitotic neurons. Proc Natl Acad Sci 96: 11317-11322.

Hartley CA, Phelps EA. 2010. Changing fear: The neurocircuitry of emotion regulation. Neuropsychopharmacology 35: 136-146.

Herrero-Mendez A, Almeida A, Fernández E, Maestre C, Moncada S, Bolaños JP. 2009. The bioenergetic and antioxidant status of neurons is controlled by continuous degradation of a key glycolytic enzyme by APC/C-Cdh1. Nat Cell Biol 11: 747-752.

Juo P, Kaplan JM. 2004. The anaphase-promoting complex regulates the abundance of GLR-1 glutamate receptors in the ventral nerve cord of $C$. elegans. Curr Biol 14: 2057-2062.

Kandel ER. 2001. The molecular biology of memory storage: A dialogue between genes and synapses. Science 294: 1030-1038.

Konishi Y, Stegmüller J, Matsuda T, Bonni S, Bonni A. 2004. Cdh1-APC controls axonal growth and patterning in the mammalian brain. Science 303: $1026-1030$.

Lee SH, Choi JH, Lee N, Lee HR, Kim JI, Yu NK, Choi SL, Lee SH, Kim H, Kaang BK. 2008. Synaptic protein degradation underlies destabilization of retrieved fear memory. Science 319: 1253-1256.
Li M, Shin YH, Hou L, Huang X, Wei Z, Klann E, Zhang P. 2008. The adaptor protein of the anaphase promoting complex Cdh1 is essential in maintaining replicative lifespan and in learning and memory. Nat Cell Biol 10: $1083-1089$.

Listovsky T, Oren YS, Yudkovsky Y, Mahbubani HM, Weiss AM, Lebendiker M, Brandeis M. 2004. Mammalian Cdh1/Fzr mediates its own degradation. EMBO J 23: 1619-1626.

Lopez-Salon M, Alonso M, Vianna MR, Viola H, Mello e Souza T, Izquierdo I, Pasquini JM, Medina JH. 2001. The ubiquitin-proteasome cascade is required for mammalian long-term memory formation. Eur J Neurosci 14: 1820-1826.

Mabb AM, Ehlers MD. 2010. Ubiquitination in postsynaptic function and plasticity. Annu Rev Cell Dev Biol 26: 179-210.

Maestre C, Delgado-Esteban M, Gomez-Sanchez JC, Bolaños JP, Almeida A. 2008. Cdk5 phosphorylates Cdh1 and modulates cyclin B1 stability in excitotoxicity. EMBO J 27: 2736-2745.

Mao SC, Lin HC, Gean PW. 2008. Augmentation of fear extinction by D-cycloserine is blocked by proteasome inhibitors.

Neuropsychopharmacology 33: 3085-3095.

Merlo E, Romano A. 2007. Long-term memory consolidation depends on proteasome activity in the crab Chasmagnathus. Neuroscience 147: $46-52$.

Minichiello L, Korte M, Wolfer D, Kühn R, Unsicker K, Cestari V, Rossi-Arnaud C, Lipp HP, Bonhoeffer T, Klein R. 1999. Essential role for TrkB receptors in hippocampus-mediated learning. Neuron 24: $401-414$.

Myers KM, Davis M. 2007. Mechanisms of fear extinction. Mol Psychiatry 12: $120-150$

Oddo S. 2008. The ubiquitin-proteasome system in Alzheimer's disease. J Cell Mol Med 12: 363-373.

Park SK, Nguyen MD, Fischer A, Luke MP, Affar B, Dieffenbach PB, Tseng HC, Shi Y, Tsai LH. 2005. Par-4 links dopamine signaling and depression. Cell 122: 275-287.

Passmore LA, Booth CR, Venien-Bryan C, Ludtke SJ, Fioretto C, Johnson LN, Chiu W, Barford D. 2005. Structural analysis of the anaphase-promoting complex reveals multiple active sites and ingights into polyubiquitylation. Mol Cell 20: 855-866.

Peleg S, Sananbenesi F, Zovoilis A, Burkhardt S, Bahari-Java S, Agis-Balboa RC, Cota P, Wittnam J, Gogul-Doering A, Opitz L, et al. 2010. Altered histone acetylation is associated with age-dependent memory impairment in mice. Science 328: 753-756.

Pesin JA, Orr-Weaver TL. 2008. Regulation of APC/C activators in mitosis and meiosis. Annu Rev Cell Dev Biol 24: 475-499.

Peters JM. 2006. The anaphase promoting complex/cyclosome: A machine designed to destroy. Nat Rev Mol Cell Biol 7: 644-656.

Radde R, Bolmont T, Kaeser SA, Coomaraswamy J, Lindau D, Stoltze L, Calhoun ME, Jäggi F, Wolburg H, Gengler S, et al. 2006. Aß42-driven cerebral amyloidosis in transgenic mice reveals early and robust pathology. EMBO Rep 7: 940-946.

Ressler KJ, Rothbaum BO, Tannenbaum L, Anderson P, Graap K, Zimand E, Hodges L, Davis M. 2004. Cognitive enhancers as adjuncts to psychotherapy: Use of D-cycloserine in phobic individuals to facilitate extinction of fear. Arch Gen Psychiatry 61: 1136-1344.

Sananbenesi F, Fischer A. 2009. The epigenetic bottleneck of neurodegenerative and psychiatric diseases. Biol Chem 390: $1145-1153$.

Sananbenesi F, Fischer A, Wang X, Schrick C, Neve R, Radulovic J, Tsai LH. 2007. A hippocampal Cdk5 pathway regulates extinction of contextual fear. Nat Neurosci 10: 1012-1019.

Sotres-Bayon F, Cain CK, LeDoux JE. 2006. Brain mechanisms of fear extinction: Historical perspectives on the contribution of prefrontal cortex. Biol Psychiatry 60: 329-336.

Tronson NC, Schrick C, Guzman YF, Huh KH, Srivastava DP, Penzes P, Guedea AL, Gao C, Radulovic J. 2009. Segregated populations of hippocampal principal CA1 neurons mediating conditioning and extinction of contextual fear. J Neurosci 29: 3387-3394.

van Roessel P, Elliott DA, Robinson IM, Prokop A, Brand AH. 2004. Independent regulation of synaptic size and activity by the anaphase-promoting complex. Cell 119: 707-718.

Wirth KG, Ricci R, Giménez-Abián JF, Taghybeeglu S, Kudo NR, Jochum W, Vasseur-Cognet M, Nasmyth K. 2004. Loss of the anaphase-promoting complex in quiescent cells causes unscheduled hepatocyte proliferation. Genes Dev 18: 847-860.

Yang Y, Kim AH, Yamada T, Wu B, Bilimoria PM, Ikeuchi Y, de la Iglesia N, Shen J, Bonni A. 2009. A Cdc20-APC ubiquitin signaling pathway regulates presynaptic differentiation. Science 326: $575-578$.

Received September 1, 2010; accepted in revised form November 9, 2010. 


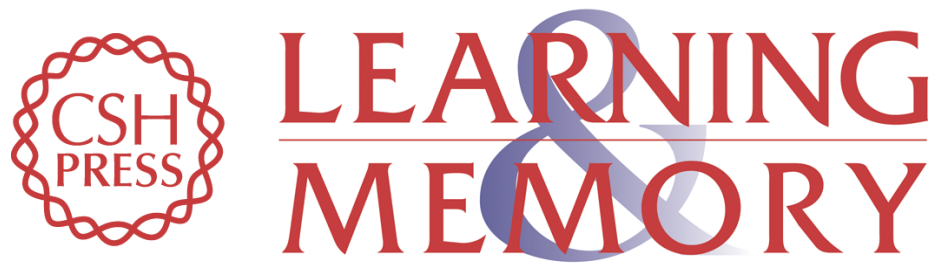

\section{The anaphase promoting complex is required for memory function in mice}

Tanja Kuczera, Roman Manuel Stilling, Hung-En Hsia, et al.

Learn. Mem. 2011, 18:

Access the most recent version at doi:10.1101/lm.1998411

Supplemental
Material http://learnmem.cshlp.org/content/suppl/2010/12/22/18.1.49.DC1

References This article cites 40 articles, 8 of which can be accessed free at: http://learnmem.cshlp.org/content/18/1/49.full.html\#ref-list-1

License

Email Alerting Receive free email alerts when new articles cite this article - sign up in the box at the Service top right corner of the article or click here. 